\title{
FPGA implementation of filtered image using 2D Gaussian filter
}

\author{
Leila kabbai *, Anissa Sghaier ${ }^{\dagger}$, Ali Douik* and Mohsen Machhout ${ }^{\dagger} *$ \\ National Engineering School of Monastir, University of Monastir Tunisia \\ $\dagger$ Faculty of sciences Monastir, University of Monastir-Tunisia \\ $\ddagger$ National Engineering School of Sousse, University of Sousse-Tunisia
}

\begin{abstract}
Image filtering is one of the very useful techniques in image processing and computer vision. It is used to eliminate useless details and noise from an image. In this paper, a hardware implementation of image filtered using 2D Gaussian Filter will be present. The Gaussian filter architecture will be described using a different way to implement convolution module. Thus, multiplication is in the heart of convolution module, for this reason, three different ways to implement multiplication operations will be presented. The first way is done using the standard method. The second way uses Field Programmable Gate Array (FPGA) features Digital Signal Processor (DSP) to ensure and make fast the scalability of the effective FPGA resource and then to speed up calculation. The third way uses real multiplier for more precision and a the maximum uses of FPGA resources. In this paper, we compare the image quality of hardware (VHDL) and software (MATLAB) implementation using the Peak Signal-to-Noise Ratio (PSNR). Also, the FPGA resource usage for different sizes of Gaussian kernel will be presented in order to provide a comparison between fixed-point and floating point implementations.
\end{abstract}

Keywords-Gaussian Filter; convolution;fixed point arithmetic;Floating point arithmetic;FPGA

\section{INTRODUCTION}

Convolution has been widely used in computer vision and image processing, including object recognition [2] and image matching [3], However, convolution operation typically requires a significant amount of computing resources [4]. Image filtering is applied as pre-processing to eliminate useless details and noise from an image. It is produced by convolution between an image and 2D Gaussian mask. In the literature, several efficient FPGA implementations of the 2D convolution operation have been proposed [5]-[9].

Hanumantharaju et al. [10] proposed a hardware architecture suitable for FPGA/ASIC implementation of a 2D Gaussian surround function for image processing application which offers a savings of memory. Barbole et al. [11] implemented steerable Gaussian smoothing filters on an FPGA platform based on a VirtexV ML506 using the pipelined approach and DSP which reduces memory requirements. Talbi et al. [5] developed architecture for separable and two-dimensional Gaussian smoothing filters, which was implemented in the VirtexV FPGA platform. They prove that the first approach is significantly faster than the second one. In the same year, Cabello et al. [2] implemented a 2D Gaussian Filter in FPGA using fixed-point arithmetic and floating point arithmetic, they found that increasing the kernel sizes, they reduced the computational costs using floating point arithmetic.

In this paper, a Gaussian filter on an Field Programmable Gate Array (FPGA) platform will be implemented. We will focus in the main bloc which is the convolution module based on the multiplication operation. Thus, the multiplier is in the heart of the proposed design. For this, the standard multiplier will be firstly implemented. Then, in order to accelerate calculus and to minimize resource use, FPGA features will be used which are DSP (Digital Signal Processor) and RAMs. Finally, in order to have more precision in image output, a real multiplier proposed in [13] will be used to implement the entire architecture. It is a new way to do a multiplication between two real numbers. Our application is implemented by two tools such as MATLAB and VHDL, and simulated on the ISE simulator.

The remainder of this paper is as follows. Section 2 introduces the image filtering algorithm. The hardware implementation of image filtering is presented in section 3. In section 4 , the hardware optimization of convolution module based on changing the multiplier will be discussed. Experimental results are given in section 5. Finally, a conclusion will be done in section 6 .

\section{IMAGE FILTERING ALGORITHM}

Smoothing filters are widely used in many applications such as object recognition, matching, classification, etc. They are applied as pre-processing for removing useless details and noise [14]. We will focus on image filtering based on Gaussian filter.

\section{A. Gaussian mask}

Gaussian filter is one of the most important and widely used filtering algorithms in image processing [5]. Gaussian filter $(G)$ is defined in equation 1 .

$$
G(x, y)=\frac{1}{2 \pi \sigma^{2}} e^{-\left(x^{2}+y^{2}\right) / 2 \sigma^{2}}
$$

where $G$ is the Gaussian mask at the location with coordinates $x$ and $y, \sigma$ is the parameter which defines the standard deviation of the Gaussian. If the value of $\sigma$ is large, the image smoothing effect will be higher. 


\section{B. Convolution operation}

In general, smoothing can be effected by convolve the original image $\mathrm{I}(\mathrm{x}, \mathrm{y})$ of the size $\mathrm{h} \mathrm{x} \mathrm{w}$ with a Gaussian mask $\mathrm{G}(\mathrm{x}, \mathrm{y})$ as illustrated in equation 2 . It is obtained by computing the sum of products among the input image and a smaller Gaussian matrix of the size $(3 \times 3)$. A 2 D convolution using a $3 \times 3$ mask and $3 \times 3$ input image is illustrated in Figure reffig1.

$$
f(x, y)=\sum_{i=0}^{h-1} \sum_{j=0}^{w-1} G(i, j) I(x-i, y-j)
$$

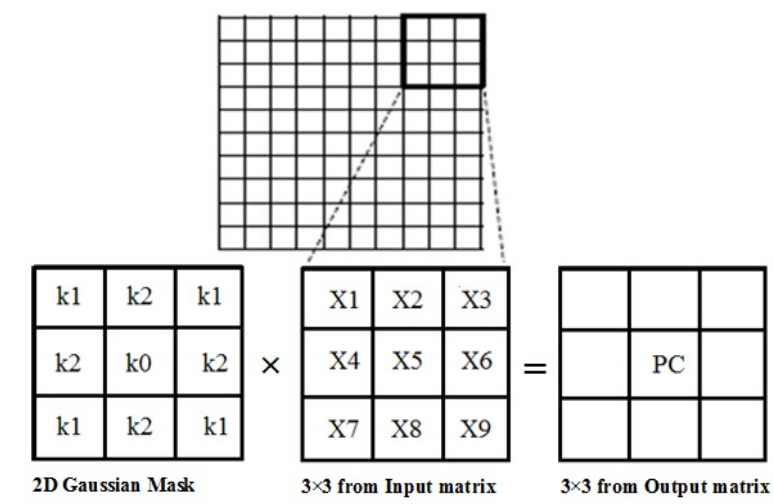

$\mathrm{PC}=\mathrm{X} 1 \times \mathrm{k} 1+\mathrm{X} 2 \times \mathrm{k} 2+\mathrm{X} 3 \times \mathrm{k} 1+\mathrm{X} 4 \times \mathrm{k} 2+\mathrm{X} 5 \times \mathrm{k} 0+\mathrm{X} 6 \times \mathrm{k} 2+\mathrm{X} 7 \times \mathrm{k} 1+\mathrm{X} 8 \times \mathrm{k} 2+\mathrm{X} 9 \times \mathrm{k} 1$

Fig. 1: Convolution operation

\section{HARDWARE IMPLEMENTATION OF IMAGE FILTERING}

In this section, the proposed architecture design of the Gaussian filter will be presented.

\section{A. Block diagram of image filtering}

Figure 2 illustrates the block diagram of image filtering. First, the input image and the Gaussian mask are read and saved by MATLAB. Next, These values are converted into a vector in a text file extension *.coe using the MATLAB tool and loaded the text file in block RAM (BRAM). The text file of Gaussian mask and image is stored respectively in BRAM1 and BRAM2. After that, the convolution operation is effected between these pixel values of two BRAM ( 1 and 2) using VHDL tool and saving the obtain results in another block (BRAM3). Finally, the text file of BRAM3 is converted by MATLAB tool in order to display the results form an image. The next step, we defined each block of diagram in Figure 2.

\section{B. Synchronous architecture hardware of image filtering}

Figure 3 depicts the block diagram of synchronous image filtering which contains a set of modules: Control Module, 3 BRAMs (matrix of input image, matrix of Gaussian mask, matrix of filtered image) and convolution Module.

\section{1) Gaussian Filter}

The convolution of an image with a Gaussian mask

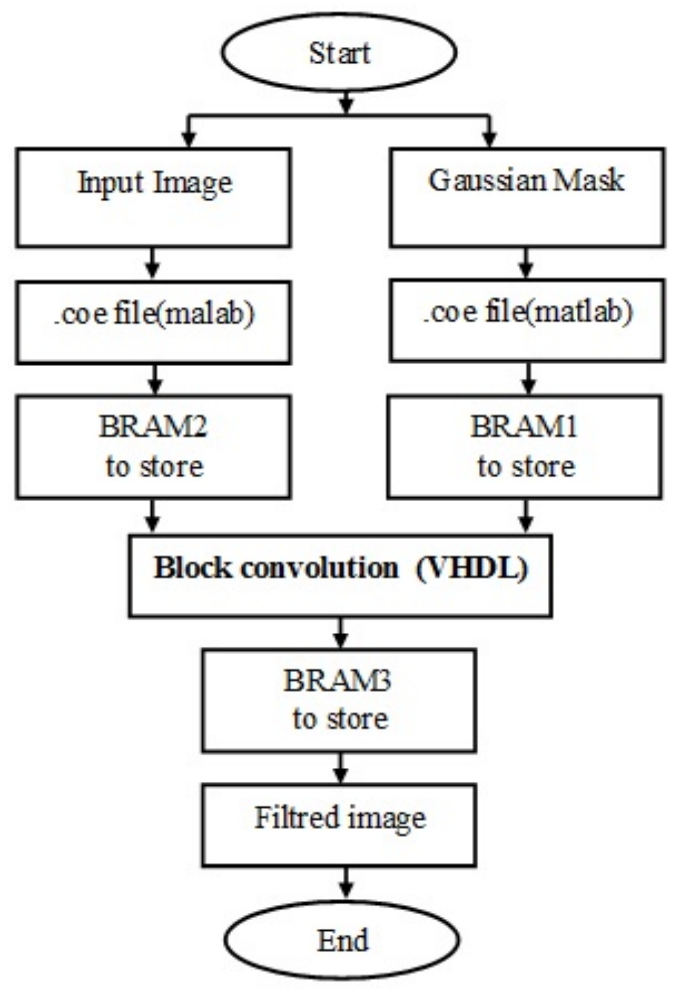

Fig. 2: Block diagram of image filtering

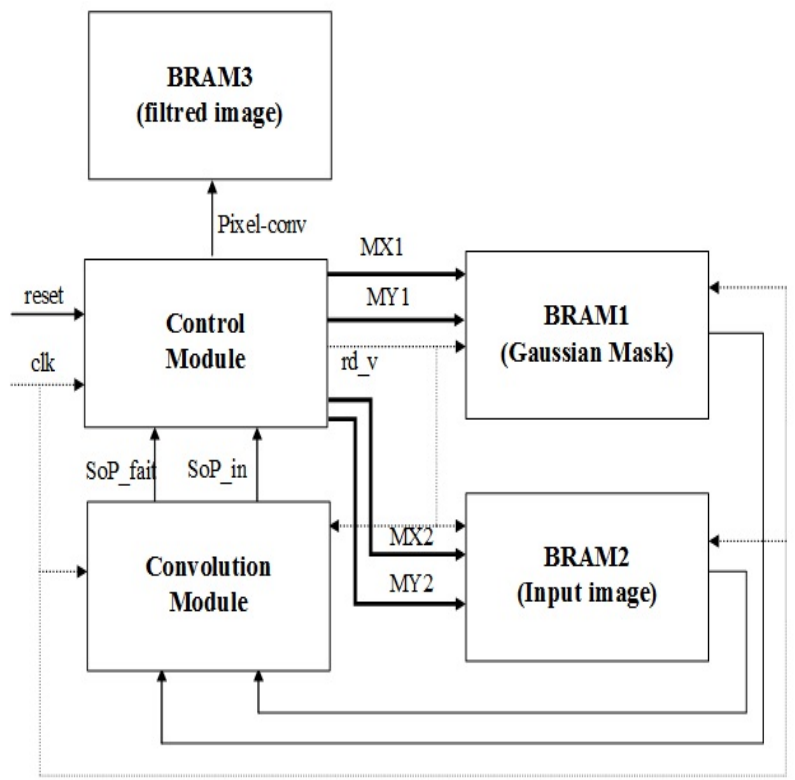

Fig. 3: Synchronous architecture of image filtering

involves floating point multiplications, which consumes considerable hardware resources. The Gaussian mask size $(3 \times 3)$ is presented by the matrix below by choosing the standard deviation equal to 0.5 .

$\left[\begin{array}{lll}0.0113 & 0.0838 & 0.0113 \\ 0.0838 & 0.6193 & 0.0838 \\ 0.0113 & 0.0838 & 0.0113\end{array}\right]$


Then, it is necessary to convert the floating point coefficients to fixed integer point coefficients for hardware implementation of the Gaussian filter. In the convolution process, each mask values has to be multiplied with each element of the image and then divided by a power of 2 [15], [16]. The approximation of the Gaussian mask is presented by equation below.

$$
\left\{\begin{aligned}
G(x, y) & =\frac{1}{2^{8}}\left[\begin{array}{ccc}
3 & 21 & 3 \\
21 & 158 & 21 \\
3 & 21 & 3
\end{array}\right] \\
& =\left[\begin{array}{ccc}
0.0117 & 0.082 & 0.0117 \\
0.082 & 0.6172 & 0.082 \\
0.0117 & 0.082 & 0.0117
\end{array}\right]
\end{aligned}\right.
$$

\section{2) Block RAM}

In Xilinx FPGAs, a Block RAM (BRAM) is a dedicated two-port memory that stores up to $36 \mathrm{~Kb}$ of data. The FPGA contains many of these blocks. Inside of each, small logic block is a configurable lookup table. It is normally used for logic functions, and it can be also reconfigured as a few bits of RAM. Several of them can be combined into a larger RAM which is denoted by a distributed RAM. BRAM is synchronous, this means that the read and write operations from and to the memory are based on the clock input signal. The read and write operations are also dependent on the read/write enable ports. In our case, BRAM2 is used to store the data test image using .coe file which is generated with Matlab tool, and a BRAM1 is used to store the .coe file of Gaussian mask, which are then read by the control module. BRAM3 will save the data filtered.

\section{3) Control module}

The control unit is an important step of the proposed synchronous architecture. It allows to generate the address to BRAMs (1 and 2) and transfers the data from each BRAM to the corresponding convolution module for computing the Sum of Products (SoP) between these values, after that the convoluted value is stored in BRAM3. The control module is designed as a Finite State Machine (FSM) simulated in VHDL. Figure 4 illustrates the Finite State Machine (FSM) of the control module.

In the first state, initialization parameter will be affected. Then in state 1, the signal rd-v will be putted to 1 to access both memories. FSM increments the counter MY1 and MY2 when the MX1 and MX2 counter are finished addressing a line of image pixel block ( 3 by 3 ) and the same Gaussian block. This process is repeated the addressing of the blocks, if it is completed then goes to state 2 if not it returns to state 1 . States 2 and 3 represent two late cycles to synchronize system signal. After that, it goes to state 4 where the machine puts the rd-v signal to zero in order to stop the addressing of the two memories and goes to state 5 . In the state 5 , the machine tests the SoP-fait signal, if it is equal to zero then it returns to the same state, if not it stored the value of SoP-in a table. After that, it increments the counter one " $i$ " or " $j "$ in order to read a new block, if " $i$ " is different to the (length of size image -1 ) and " $\mathrm{j}$ " is different (width of size image -1 ) then returns to

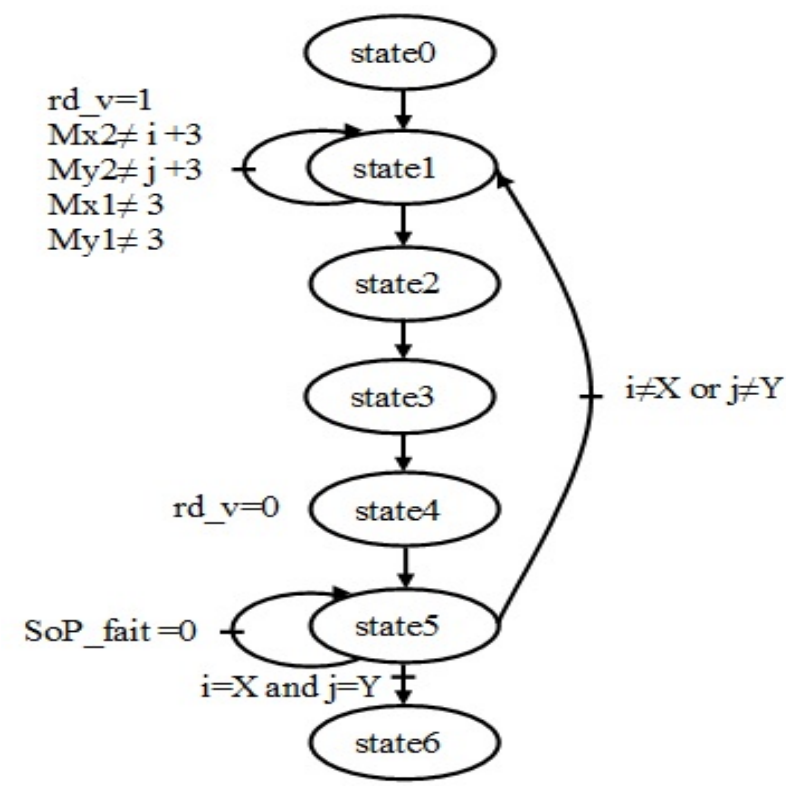

Fig. 4: FSM of the control unit

state 1 . If not goes to state 6 (end process). Where, $X$ is the length of size image -1 and $Y$ is the width of size image -1 .

4) Convolution Module

Convolution module focuses on the calculation of the sum of products (SoP) between pixels in BRAM1 and BRAM2 for a window of 3 by 3 . Equation 5 depicts an example of the convolution module between Gaussian mask integer and matrix $3 \times 3$ from input image.

\section{HARDWARE OPTIMIZATION OF CONVOLUTION MODULE}

The main operation in the convolution module is the multiplication.

\section{A. Convolution module using standard method}

Signal multiplication of $3 \times 3$ image by $3 \times 3$ mask will be done. Multiplier input values are loaded into the RAM block addresses port registers (the outputs of the RAM blocks (BRAM1 and BRAM2) are the inputs of the multiplier). One multiplication is completed in one clock cycle, thus the other 9 multiplications will take 9 clock cycles. The partial product of each multiplication will be summed to obtain the final result. The final result of the multipliers will be stored also in RAM blocks (BRAM 3).

\section{B. Convolution module using FPGA multiplier}

FPGA devices have dedicated architectural features that make it easy to implement high performance multipliers. FPGA devices feature embedded high-performance multiplieraccumulators (MACs) in dedicated Digital Signal Processor (DSP) blocks. For high performance applications, DSP blocks can speedup different operations. Embedded multiplier blocks using DSP will be used in our Gaussian filter for low cost and 
speedup smoothing image.

These multipliers are implemented in a combination of DSP blocks or embedded multipliers and logic resources. DSP is a multiplication-intensive technology and to achieve high speeds, these multiplication operations must be accelerated. The base of many DSP algorithms is multiplication. In this operation, each element of the multiplier is multiplied by each bit of the multiplicand. Then, the partial product of each multiplication is accumulated according to the weight of the partial product, where the weight indicates the location of a bit corresponding to other bits.

- Multiplication: Multiple memory blocks produce one multiplication result every clock cycle. This mode is useful for high-speed data scaling.

- Sum of multiplication: One memory block or group of memory blocks produces the sum of multiplication results.

\section{Convolution module using real multiplier}

From a practical point of view, using floating point multiplications to calculate the convolution can consumes considerable hardware resources but it offers more precision and a good smoothing of the input image. In this section, we will present the used multiplier proposed in [13]. Hence, if we want to multiply real coefficients like this example: $a=3,14$ and $b=4,15$. We may first define $\alpha_{a}$ and $\alpha_{b}$ which are number of terms after comma. In our example: $\alpha_{a}=2$ and $\alpha_{b}=2$. And, we divide $a$ and $b$ into two parts:

$$
\left\{\begin{array}{l}
X_{a}=3 \text { and } Y_{a}=14 \\
X_{b}=4 \text { and } Y_{b}=15
\end{array}\right.
$$

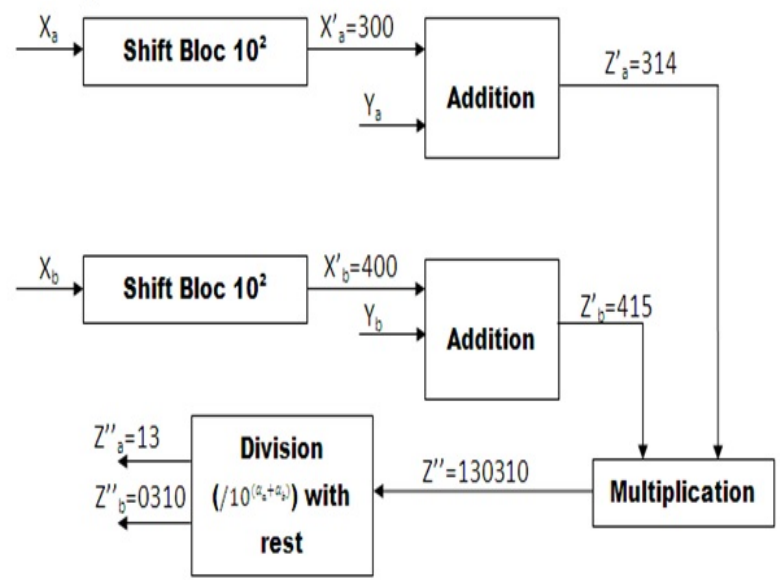

Fig. 5: Proposed Multiplication architecture

In Figure 5, the first part of $a$ should be multiplied by $10^{\alpha_{a}}$ and $b$ by $10^{\alpha_{b}}$. To ensure the multiplication we can use the left shifting function. Then we add the result to the correspondent second part of $a$ and $b$, so we will have $Z_{a}^{\prime}$ and $Z_{b}^{\prime}$. Then after multiplying these two terms we have to divide the result by $10^{\left(\alpha_{a}+\alpha_{b}\right)}$. To implement this division, we can use right shifting function. The final result is: $R=a \times b=13,0310$.

\section{EXPERIMENTAL RESULTS}

In this section, simulations and implementation results will be discussed.

\section{A. Performance Measures}

The Peak Signal to Noise Ratio (PSNR) is the most used parameter to evaluate image quality in the literature [11], [17][20], [22]. PSNR value can be computed by comparing two images which are original image and filtered image. The PSNR was used to measure the image quality. A higher PSNR value indicates that the filtered image contains better image quality. The PSNR has been calculated as follows;

$$
P S N R=10 \log _{10}\left(\frac{255^{2}}{M S E}\right)
$$

Where, MSE is the Mean Square Error (equation 4) between the original image $(\mathrm{I} 1(\mathrm{~m}, \mathrm{n}))$ and the filtered image ( $I 2(m, n))$, with, $m$ and $n$ are pixels of image $M N$.

$$
M S E=\frac{1}{M \times N} \sum_{m=1}^{M} \sum_{n=1}^{N}\left(I_{1}(m, n)-I_{2}(m, n)\right)^{2}
$$

\section{B. Simulation results in MATLAB and VHDL}

In this section, simulation and implementation results will be done. Figure 6 presents the filtered image by two tools which are MATLAB and ModelSim-SE (VHDL).

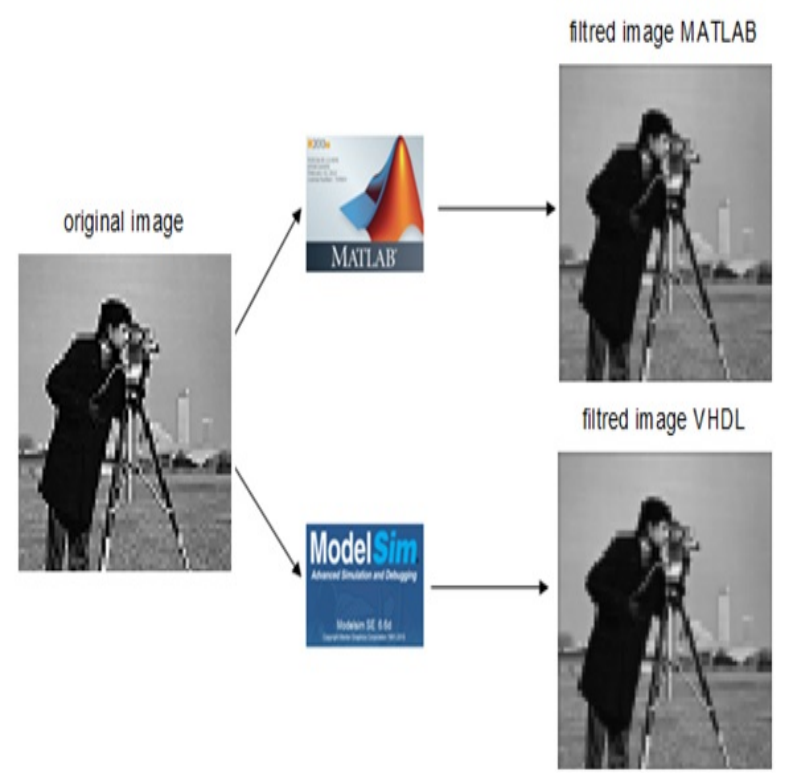

Fig. 6: Resulting filtered image in both MATLAB and VHDL

The kernel size $3 \times 3$ will be conserved and sigma values will be changed in order to see their impact in the filtered image. Figure 7, 8 and 9 illustrate the filtered image by the software (MATLAB) and hardware (VHDL) implementations. We can deduce that the blurring effect increases proportional to the sigma value (respectively $0.5,1$ and 1.5).

For different sigma values, Table I resumes the corresponding PSNR of images (in both VHDL and MATLAB). 
original image

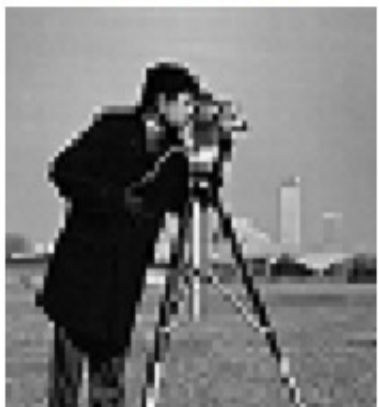

filtred image MATLAB

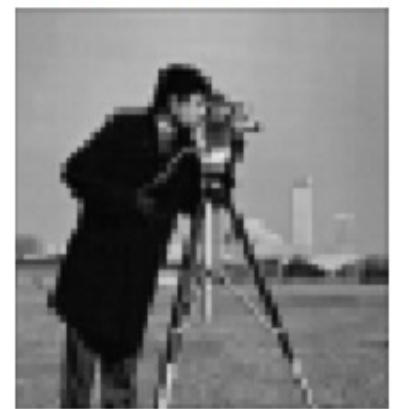

filtred image VHDL

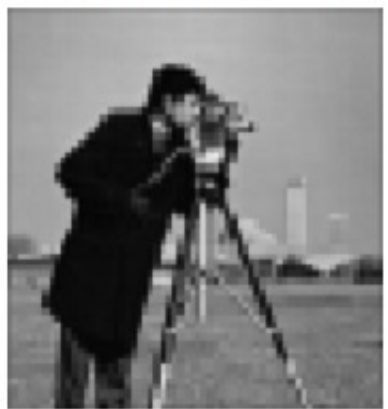

Fig. 7: Filtered image with sigma $=0.5$

filtred image MATLAB

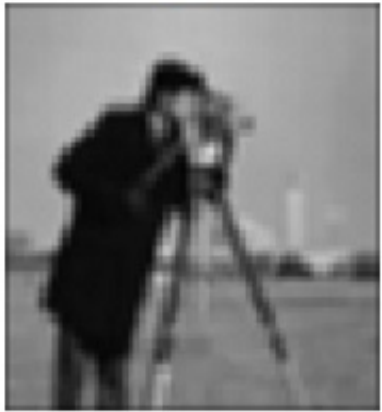

filtred image VHDL

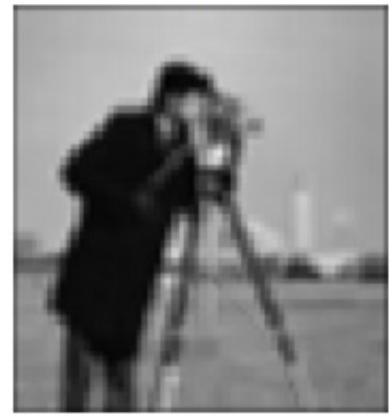

Fig. 8: Filtered image with sigma=1

\section{filtred image MATLAB}

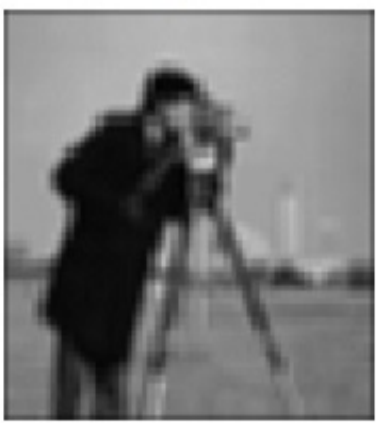

filtred image VHDL

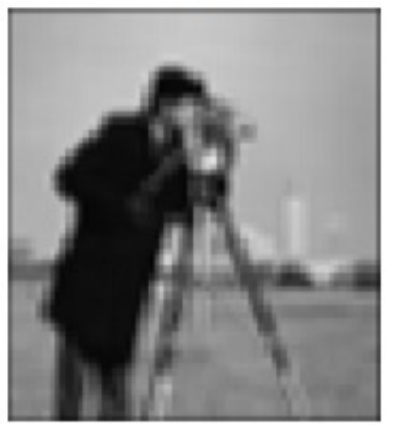

Fig. 9: Filtered image with sigma $=1.5$

For sigma equal 0.5, we observe that the PSNR (VHDL) obtains better result compared to PSNR (MATLAB). So, when increase sigma, the PSNR value of MATLAB and VHDL are decreased. Figure 10 shows the comparison between PSNR values both resulting image in MATLAB and VHDL.

Normally, if PSNR value is more than $40 \mathrm{~dB}$, this is an indication that the quality of the image is good. But, if the image is mean quality, the PSNR value is less than $30 \mathrm{db}$ which is the case of our selected image. We note that when we vary the sigma value the effect of smoothing increase and the PSNR decrease.
TABLE I: PSNR values for different output images in VHDL and MATLAB

\begin{tabular}{|c|c|c|}
\hline & PSNR (MATLAB) & PSNR (VHDL) \\
\hline Sigma $=0.5$ & 25.2236 & 27.3294 \\
\hline Sigma $=1$ & 19.8879 & 20.3760 \\
\hline Sigma $=1.5$ & 18.0441 & 19.6098 \\
\hline
\end{tabular}

\section{Simulation results and resources utilization}

Modern FPGA families integrate many features into the silicon. These features reduce the area required and increase 


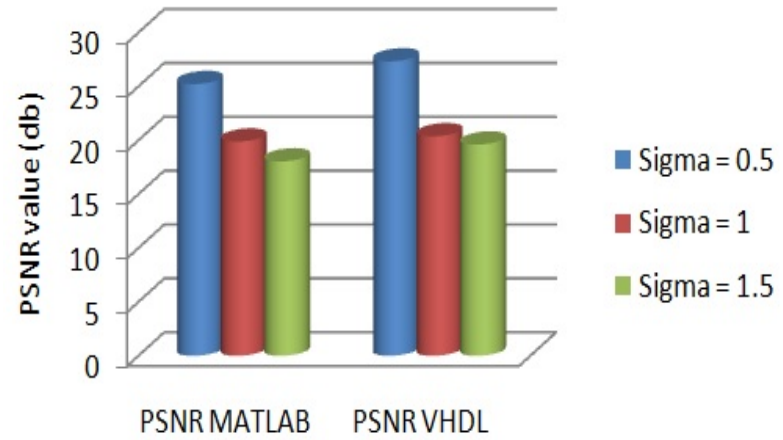

Fig. 10: Comparison between PSNR values to both resulting image in MATLAB and VHDL

speed compared to building them from primitives. For examples: multipliers, generic DSP blocks, embedded processors, high speed I/O logic and embedded memories. To ensure the correctness of the proposed architecture, the algorithm of Gaussian filter has been firstly coded and tested in MATLAB (Version 12.1), then an FPGA implementation was coded in RTL compliant VHDL and the hardware simulation results have been obtained using ModelSim (Version SE 6.4) and synthesized using Xilinx ISE 12.4.

The proposed design has been implemented on Xilinx VirtexV device. In the proposed work, Gaussian design is generic, thus it can be upgraded to any size without an appreciable increase in the hardware. Hence, the functional modules are control module, BRAM, multiplier and adder. Here, experiments are performed on image of size $8 \times 8$ using 2D Gaussian mask of $3 \times 3$. The simulation results of convolution are shown in Figure 11 and device utilization summary of the implementation is given in Table II, III, and IV.

Figure 11 presents the addressing of the two blocks (BRAM1 et BRAM2) and the result obtained by calculation the Sum of Products (SoP) between pixels in BRAM1 and BRAM2 using VHDL tool.

TABLE II: Performance comparison with the state of the art implementations

\begin{tabular}{|c|c|c|c|}
\hline & Slices Registers & Slices LUTs & DSP48Es \\
\hline Ours & 127 & 176 & 9 \\
\hline 2D [5] & 228 & 2089 & 6 \\
\hline$[23]$ & 369 & 480 & - \\
\hline
\end{tabular}

Comparing our results to those in [5], we note that we decrease the number of slice registers by $44.3 \%$ and by $65.5 \%$ compared to [23]. Table III compares the results of both fixed point arithmetic and floating point arithmetic. As we can see, we decrease the number of slice registers and slice LUTs comparing to [12].

In addition, we note that floating arithmetic uses more than fixed one but still little compared to the state of the art and we should not forget that it gives more precision to filtered image.
TABLE III: Comparing fixed arithmetic results to floating arithmetic one

\begin{tabular}{|c|c|c|}
\hline & Slices Registers & Slices LUTs \\
\hline Fixed Arithmetic (ours) & 127 & 176 \\
\hline Float Arithmetic (ours) & 138 & 3080 \\
\hline Fixed Arithmetic [12] & 135 & 209 \\
\hline Float Arithmetic [12] & 151 & 5052 \\
\hline
\end{tabular}

If we increase kernel size we obtain results in Table IV. As we can see, kernel size have a big influence in design performances so that area occupation increase by the increase of kernel size.

Our results outperform those in [12] in term of slices registers and LUTs by $6 \%$ and $15 \%$ for fixed arithmetic using kernel

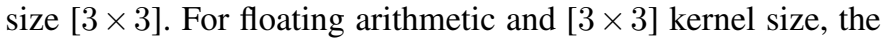
area use decrease by $8 \%$ and $39 \%$ in term of slices registers and LUTs. It is the same to the other kernel sizes.

\section{CONCLUSION}

Hardware implementation of the Gaussian filter is faster than software one. Thus, using FPGA we are able to process the filtering at the same time of reading the image. In this paper, we have presented the implementation of two-dimensional convolution on a Xilinx VirtexV FPGA platform based on a state machine. We implemented Gaussian filters with different sigma values. Then we optimized the proposed architecture using different multipliers. At the first, we used the standard multiplication " $\times$ " used in VHDL language. Then we explored FPGA features and DSP blocks. Finally, we introduced floating point arithmetic. Performances and results show that area and resources utilization decrease specially when using DSP and BRAM of FPGA. Also, speed increase comparing to the other solutions. By using floating point arithmetic the image has more precision and result seems to be is better.

\section{REFERENCES}

[1] H. Kopka and P. W. Daly, A Guide to $B T_{E} X$, 3rd ed. Harlow, England: Addison-Wesley, 1999.

[2] DG. Lowe, Distinctive image features from scale-invariant keypoints, International Journal of Computer Vision; 60(2), pp. 91-110, 2004.

[3] L. Kabbai, M. Abdellaoui, A. Douik, New robust descriptor for image matching, Journal of Theoretical and Applied Information Technology, 87(3), pp. 451- 460, 2016.

[4] L. Rao, B. Zhang, J. Zhao, Hardware Implementation of Reconfigurable ID Convolution, Journal of Signal Processing Systems, 82(1), pp. 1-16, 2016.

[5] F.Talbi, F.Alim, S. Seddiki, I. Mezzah, B. Hachemi, Separable Convolution Gaussian Smoothing Filters on a Xilinx FPGA platform, International conference on innovative computing technology (INTECH), Galcia, pp.112-117, May 2015.

[6] M.Neggazi, M.Bengherabi, A.Amira, Z.Boulkenafet, An Efficient FPGA Implementation of Gaussian Mixture Models Based Classifier, IEEE.International Workshop on Systems, Signal Processing and their Applications (WoSSPA), Algiers , pp. 367-371.May 2013.

[7] H. Zhang, M. Xia, and G. Hu, A Multiwindow partial buffering scheme for FPGA based 2-D convolvers, IEEE Transactions on Circuits and Systems II: Express Briefs, 54(2), pp. 200 - 204, February 2007.

[8] L. Chang, J. Hernndez Palancar, L.E. Sucar, M. Arias-Estrada, FPGAbased detection of SIFT interest key points, Machine vision and applications, 24(2), pp.371-392, 2013. 

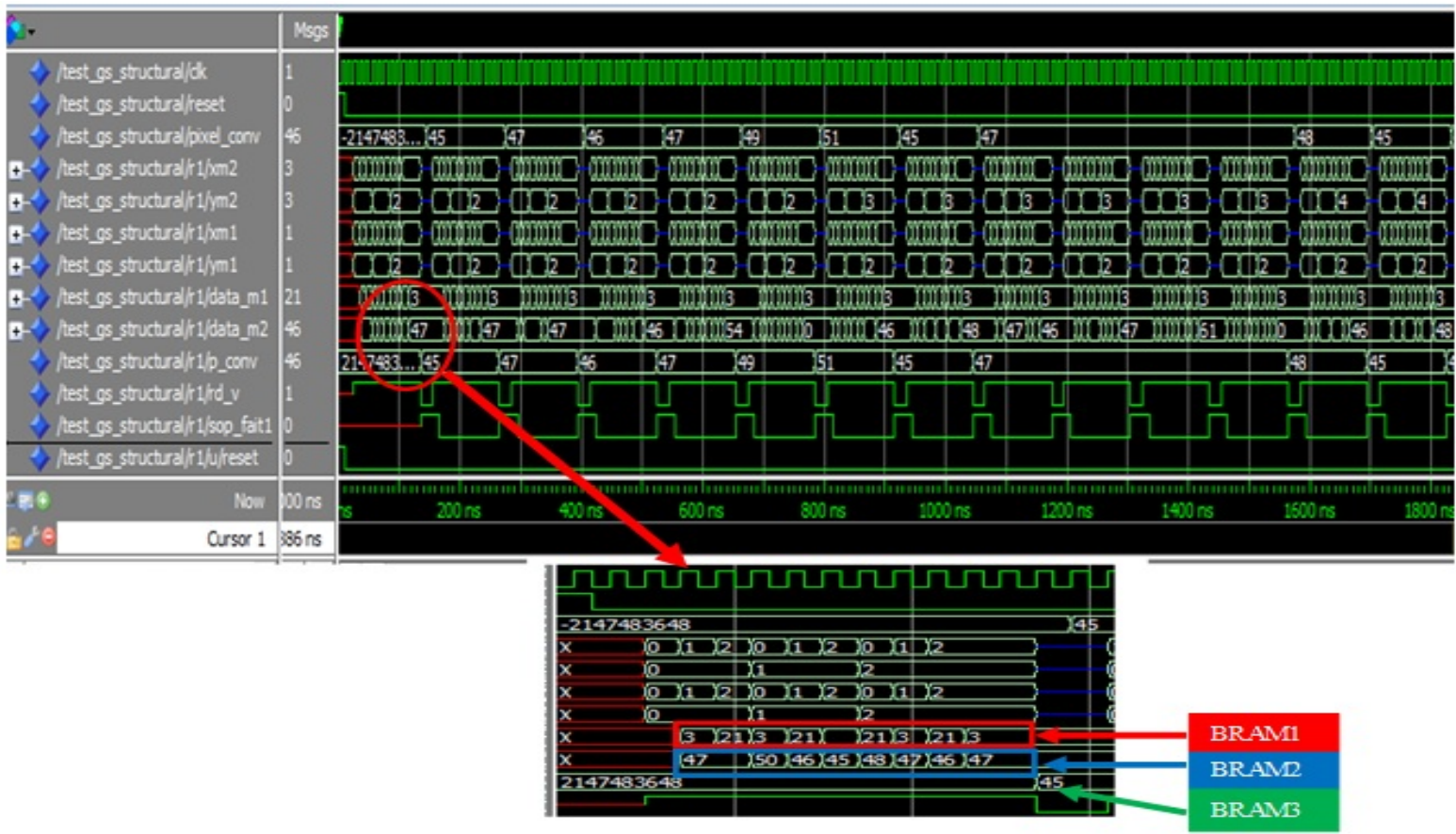

Fig. 11: Simulation results of two-dimensional convolution method

TABLE IV: Implementation results for different kernel sizes

\begin{tabular}{|c|c|c|c|c|c|c|}
\hline & \multicolumn{6}{|c|}{ kernel size } \\
\hline & \multicolumn{2}{|c|}{$[3 \times 3]$} & \multicolumn{2}{|c|}{$[5 \times 5]$} & \multicolumn{2}{|c|}{$[7 \times 7]$} \\
\hline & Unsigned & Float & Unsigned & Float & Unsigned & Float \\
\hline Slices Registers(ours) & 127 & 138 & 378 & 579 & 546 & 774 \\
\hline LUTs & 176 & 3080 & 1270 & 14559 & 20380 & \\
\hline Slices Registers [12] & 135 & 151 & 1181 & 583 & 1687 & 883 \\
\hline LUTs & 209 & 5052 & 2296 & 32557 & 2626 & 54988 \\
\hline
\end{tabular}

[9] V. Bonato, E. Marques, G. A Constantinides, A parallel hardware architecture for scale and rotation invariant feature detection, Circuits and Systems for Video Technology, IEEE Transactions on, 18(12), pp.1703-1712. 2008

[10] M. C Hanumantharaju, M. Ravishankar, D. R Rameshbabu, Design and FPGA Implementation of an 2D Gaussian Surround Function with Reduced On-Chip Memory Utilization, IEEE International Conference on Advances in Computing, Communications and Informatics (ICACCI), Mysore, pp. 604 - 609, August 2013

[11] S. Barbole and S. Shah, Efficient Pipelined FPGA Implementation of Steerable Gaussian Smoothing Filter, International Journal of Science and Research, 3(8), pp.1753-1758, 2014.

[12] Frank Cabello, Julio Leon, Yuzo lana, Rangel Arthur: Implementation of a Fixed-Point 2D Gaussian Filter for Image Processing based on FPGA, Signal Processing: Algorithms, Architectures, Arrangements, and Applications (SPA), Poznan, pp.28 - 33, September, 2015

[13] A. Sghaier, M. Zeghid, M. Machhout, Proposed efficient arithmetic operations architectures for Hyperelliptic Curves Cryptosystems (HECC), The International Multi-Conference on Systems, Signals and Devices, Mahdia, pp.1-5, March 2015.

[14] S. Rashid, S. R. Dixit, A. Y. Deshmukh, VHDL Based Canny Edge Detection Algorithm, International Journal of Current Engineering and Technology, 4(2), pp.2277, 4106, 2014.

[15] M. N. Alsharif, Real Time Image Processing for Lane Following, Master Thesis, 2014.

[16] N. S. Tahiyah, P. Vikramkumar, K. Sridharan, T.Vineetha, J. Arthi, Very large-scale integration architecture for video stabilisation and implementation on a field programmable gate array-based autonomous vehicle, IET Computer Vision, 9(4), pp. 559-569,2015.

[17] B. Sankur, K. Sayood, I. Avcibas, Statistical evaluation of Image quality measure, Journal of Electronic Imaging, 11(2), pp.206-223, 2002.

[18] M. Carnec, Critre de qualit d'images couleur avec rfrence rduite perceptuelle gnrique, Polytechnique de Nantes, These, 2004.

[19] C. Delgeorge, C. Rosenberger G. Poisson, P. Vieyres, Towards a new tool for the evaluation of the quality of Ultrasound compression Images, IEEE transactions on Mdical Imaging, 25(11), pp.1502-1509, 2006.

[20] P. Marziliano, F. Dufaux, S. Winkler, T.Ebrahimi, Perceptual blur and ringing metrics : application to jpeg 2000, Signal Processing : Image communication, 19(2), pp. 163-172, 2004

[21] J.L. Olives, Optimisation globale d'un systme imageur l'aide de critres de qualit visuelle. Ecole nationnale suprieur de l'aronautique et de l'espace, 1998.

[22] B. Rajan, S. Ravi, FPGA based hardware implementation of image filter with dynamic reconfiguration architecture, IJCSNS International Journal of Computer Science and Network Security, 6(12), pp. 121-127, 2006.

[23] S. Eswar, Noise reduction and image smoothing using gaussian blur, Masters of Science in Electrical engineering, California State University, Northridge, 2015. 